LAPTH-1042/04

\title{
Non-asymptotically flat, non-AdS dilaton black holes
}

\author{
Gérard Clément*and Cédric Leygnac ${ }^{\dagger}$ \\ Laboratoire de Physique Théorique LAPTH (CNRS), \\ B.P.110, F-74941 Annecy-le-Vieux cedex, France
}

\begin{abstract}
We show that previously known non-asymptotically flat static black hole solutions of Einstein-Maxwell-dilaton theory may be obtained as near-horizon limits of asymptotically flat black holes. Specializing to the case of the dilaton coupling constant $\alpha^{2}=3$, we generate from the non-asymptotically flat magnetostatic or electrostatic black holes two classes of rotating dyonic black hole solutions. The rotating dyonic black holes of the "magnetic" class are dimensional reductions of the five-dimensional Myers-Perry black holes relative to one of the azimuthal angles, while those of the "electric" class are twisted dimensional reductions of rotating dyonic Rasheed black strings. We compute the quasi-local mass and angular momentum of our rotating dyonic black holes, and show that they satisfy the first law of black hole thermodynamics, as well as a generalized Smarr formula. We also discuss the construction of non-asymptotically flat multi-extreme black hole configurations.
\end{abstract}

\footnotetext{
*Email: gclement@lapp.in2p3.fr

†Email: leygnac@lapp.in2p3.fr
} 


\section{Introduction}

The investigation of black hole properties in general relativity has long been restricted to the case of asymptotically flat or asymptotically (anti-)de Sitter ((A)dS) black holes. To our knowledge, non-asymptotically flat, non-AdS black holes in four (and higher) dimensions were first found in 11 as spherically symmetric solutions to Einstein-Maxwell-dilaton theory. The properties of such non-asymptotically flat solutions to Einstein-Maxwell-dilatonaxion theory in four dimensions were extensively studied in 2]. Similar nonasymptotically flat topological black holes in four dimensions were found in [3, and extended to higher dimensions in 4]. The aim of the present work is, first to reinvestigate four-dimensional non-asymptotically flat, spherically symmetric dilaton black holes for general dilatonic coupling, second to analyze in more detail the special case with dilaton coupling constant $\alpha^{2}=3$.

A motivation to investigate non-asymptotically flat, non-AdS black holes is that these might lead to possible extensions of AdS/CFT correspondence. Indeed, it has been speculated that the linear dilaton spacetimes, which arise as near-horizon limits of dilatonic black holes, might exhibit holography 5 . Another motivation is that such solutions may be used to extend the range of validity of methods and tools originally developed for, and tested in the case of, asymptotically flat or asymptotically AdS black holes. Specifically, we shall show that the quasi-local energy approach [6, 7, 8, 9] may be applied successfully to the computation of the mass and angular momentum of nonasymptotically flat rotating black holes. We shall also show that such black holes follow the first law of black-hole thermodynamics, originally formulated in the case of asymptotically flat black holes [10].

In the next section we recover the non-asymptotically flat (NAF) static black hole solutions of Einstein-Maxwell-dilaton (EMD) theory as near-horizon, near-extreme limits of asymptotically flat black holes, and we discuss briefly their properties. Section 3 is devoted to multicenter solutions of EMD; these are found to fall in two classes, one of which includes both asymptotically flat and NAF multi-extreme black holes. The computation of the quasilocal mass of the NAF static black holes is recalled in Sect. 4.

The case with dilaton coupling constant $\alpha^{2}=1$ has been considered in more detail in 2], where the NAF static black holes have been extended to rotating black hole solutions of Einstein-Maxwell-dilaton-axion gravity (EMDA). Here we shall consider the case $\alpha^{2}=3$, which is a dimensional reduction of five-dimensional Kaluza-Klein theory. Unlike the case of four- 
dimensional general relativity, the five-dimensional vacuum Einstein equations admit solutions with event horizons of various topologies [11: the static Gibbons-Wiltshire black string (topology $S^{2} \times R$ ) family [12, extended to rotating black strings by Rasheed [13]; the static Tangherlini black hole (topology $S^{3}$ ) [14, extended to rotating black holes by Myers and Perry [15]; and the Emparan-Reall rotating black rings (topology $S^{2} \times S^{1}$ ) [16. These are all asymptotically flat in five-dimensions. The NAF $\left(\alpha^{2}=3\right)$ EMD static black holes and their rotating and dyonic generalizations will turn out to correspond to special dimensional reductions of these asymptotically flat five-dimensional black holes.

In Sect. 5, we use the group $S L(3, R)$ of invariance transformations of the stationary sector of $\left(\alpha^{2}=3\right)$ EMD to generate NAF rotating magnetic black holes. We find that these are a dimensional reduction of a subclass of Myers-Perry black holes. Conversely, we find in Sect. 6 that the dimensional reduction of the generic Myers-Perry black hole depending on three parameters (mass and two angular momenta) leads to a wider class of NAF rotating dyonic black holes. In the extreme case, these may be further generalized to regular multicenter configurations. We then compute the quasilocal mass and angular momentum of our rotating dyonic black holes, and check the validity of the generalized first law of thermodynamics [13. Finally, we use in Sect. 7 electromagnetic duality to generate from this "magnetic" family a dual "electric" sector of NAF rotating dyonic black holes, which are found to correspond to a twisted dimensional reduction of Rasheed rotating dyonic black strings with a NUT charge balancing the magnetic charge. We conclude in Sect. 8.

\section{Static NAF dilaton black holes}

Consider EMD theory, defined by the action

$$
S=\frac{1}{16 \pi} \int d^{4} x \sqrt{|g|}\left\{R-2 \partial_{\mu} \phi \partial^{\mu} \phi-\mathrm{e}^{-2 \alpha \phi} F_{\mu \nu} F^{\mu \nu}\right\}
$$

where $F=d \mathcal{A}$, and $\alpha$ is the dilaton coupling constant. Special values of $\alpha$ are $\alpha=0$, corresponding to Einstein-Maxwell theory, $\alpha=1$, which is a truncation of the bosonic sector of $D=4, \mathcal{N}=4$ supergravity, and $\alpha=\sqrt{3}$, which corresponds to a dimensional reduction of five-dimensional Einstein gravity with a spacelike Killing vector (Kaluza-Klein theory). The 
static, spherically symmetric, asymptotically flat black hole solutions of EMD were found in [17] and rediscovered in [18. They exist in two versions, electrostatic or magnetostatic, related to each other by the electro-magnetic duality transformation

$$
(\phi, F) \rightarrow\left(\hat{\phi}=-\phi, \hat{F}= \pm \mathrm{e}^{-2 \alpha \phi} \tilde{F}\right),
$$

with $^{1} \tilde{F}^{\mu \nu}=\frac{1}{2} E^{\mu \nu \lambda \tau} F_{\lambda \tau}$. The electric black hole solutions are

$$
\begin{aligned}
d s^{2}= & -\frac{\left(r-r_{-}\right)^{\gamma}\left(r-r_{+}\right)}{r^{1+\gamma}} d t^{2}+ \\
& \quad+\frac{r^{1+\gamma}}{\left(r-r_{-}\right)^{\gamma}\left(r-r_{+}\right)}\left[d r^{2}+\left(r-r_{-}\right)\left(r-r_{+}\right) d \Omega^{2}\right] \\
F= & \frac{Q e^{2 \alpha \phi_{\infty}}}{r^{2}} d r \wedge d t, \quad e^{2 \alpha\left(\phi-\phi_{\infty}\right)}=\left(1-\frac{r-}{r}\right)^{1-\gamma},
\end{aligned}
$$

with

$$
\gamma=\frac{1-\alpha^{2}}{1+\alpha^{2}}
$$

The black hole parameters $r_{+}$(the location of the event horizon) and $r_{-}$(for $\alpha \neq 0$, the spacelike singularity, $\left.0<r_{-}<r_{+}\right)$are related to the physical parameters $\mathcal{M}$ (mass) and $Q$ (electric charge) by

$$
\mathcal{M}=\frac{r_{+}+\gamma r_{-}}{2}, \quad Q=e^{-\alpha \phi_{\infty}} \sqrt{\frac{1+\gamma}{2}} \sqrt{r_{+} r_{-}} .
$$

The solutions (2.3) have been proved to be the only electrostatic, asymptotically flat regular black hole solutions for $\alpha=1$ [19] and, more recently, for arbitrary $\alpha$ [20]. However this proof leaves open the possibility, for $\alpha \neq 0$, of NAF electrostatic black holes, generalizing the $\alpha=1$ linear dilaton black holes [21, 2]. These have previously been found by a direct solution of the field equations of EMD [1] (see also [22, 23]). Here we shall recover these non-asymptotically flat black holes by taking the near-extreme, near-horizon limit of the asymptotically flat solutions (2.3). Define three new black-hole parameters $r_{0}, b$ and $\nu$ by

$$
r_{-}=\epsilon^{-\alpha^{2}} r_{0}, \quad r_{+}=\epsilon^{-\alpha^{2}} r_{0}+\epsilon b, \quad \phi_{\infty}=\alpha^{-1} \ln \nu-\alpha \ln \epsilon,
$$

\footnotetext{
${ }^{1}$ Here $E^{\mu \nu \lambda \tau} \equiv|g|^{-1 / 2} \varepsilon^{\mu \nu \lambda \tau}$, with $\varepsilon^{1234}=+1$, where $x^{4}=t$ is the time coordinate.
} 
where the dimensionless parameter $\epsilon$ shall eventually be taken to zero, and transform the $(t, r)$ coordinates to

$$
t=\epsilon^{-1} \bar{t}, \quad r=\epsilon^{-\alpha^{2}} r_{0}+\epsilon \bar{r} .
$$

Finally take the limit $\epsilon \rightarrow 0$ and relabel $\bar{t} \rightarrow t, \bar{r} \rightarrow r$, which yields

$$
\begin{aligned}
d s^{2} & =-\frac{r^{\gamma}(r-b)}{r_{0}^{1+\gamma}} d t^{2}+\frac{r_{0}^{1+\gamma}}{r^{\gamma}(r-b)}\left[d r^{2}+r(r-b) d \Omega^{2}\right] \\
F & =\sqrt{\frac{1+\gamma}{2}} \frac{\nu}{r_{0}} d r \wedge d t, \quad e^{2 \alpha \phi}=\nu^{2}\left(\frac{r}{r_{0}}\right)^{1-\gamma} .
\end{aligned}
$$

The magnetic dual version also exists, with the same metric supported by the magnetic and dilaton fields

$$
F=\sqrt{\frac{1+\gamma}{2}} \frac{r_{0}}{\nu} \sin \theta d \theta \wedge d \varphi, \quad e^{2 \alpha \phi}=\nu^{-2}\left(\frac{r}{r_{0}}\right)^{\gamma-1} .
$$

These NAF solutions of EMD depend on three parameters, $\nu$ which accounts for the invariance under dilaton rescalings $\phi \rightarrow \phi+$ constant, $r_{0}>0$ which sets the overall scale and is related to the electric charge $Q$ according to

$$
Q=\frac{1}{4 \pi} \int \mathrm{e}^{-2 \alpha \phi} F^{0 r} \sqrt{|g|} d \Omega=\sqrt{\frac{1+\gamma}{2}} \frac{r_{0}}{\nu},
$$

and the horizon radius $b$, which we expect to be proportional to the black hole mass $\mathcal{M}$, the exact computation (recalled in Sect. 4) giving [1]

$$
\mathcal{M}=\frac{(1-\gamma) b}{4}
$$

For $\alpha^{2}=0(\gamma=1)$, the family of solutions (2.8)-(2.10) depending on $b$ correspond to different parametrizations of the Bertotti-Robinson spacetime $A d S_{2} \times S^{2}$ generated by a homogeneous electric or magnetic field. Being related to the $b=0$ solution by global coordinate transformations, these solutions are not black holes, and accordingly their mass vanishes. For $\alpha^{2} \rightarrow \infty(\gamma=-1)$, the dilaton and electromagnetic fields decouple and the solutions (2.8) reduce to the Schwarzschild black holes with the appropriate mass $\mathcal{M}=b / 2$. Thus, this family of non-asymptotically flat black hole solutions interpolate continuously between the Bertotti-Robinson and Schwarzschild solutions. 
The Penrose diagrams corresponding to the different values of $b(b<0$, $b=0$ and $b>0)$ are shown in Fig. 1 for $0<\alpha^{2}<1(0<\gamma<1)$, Fig. 2 for $\alpha^{2}=1(\gamma=0)$ and Fig. 3 for $\alpha^{2}>1(\gamma<0)$. The Ricci scalar

$$
R=-\frac{1-\gamma^{2}}{2} \frac{(r-b) r^{\gamma-2}}{r_{0}^{1+\gamma}}
$$

vanishes at spatial infinity $r \rightarrow \infty$ and on the horizon, and is singular for $r=0$. For $\alpha^{2}<1$, spatial infinity is conformally time-like, as in the $A d S$ case, leading to a Penrose diagram for the black-hole case $b>0$ which is similar to that of the three-dimensional BTZ static black hole [24]. For $\alpha^{2} \geq 1$, spatial infinity is conformally null, with a Schwarzschild-like Penrose diagram in the black-hole case. The extreme black holes $(b=0)$ are all singular, the singularity being null for $\alpha^{2} \leq 1$ and timelike for $\alpha^{2}>1$.

\section{Extreme case: multicenter solutions}

As in the special case $b=0$ of the linear dilaton [2], the extreme $b=0$ solutions have a conformally flat spatial metric, which suggests that they can be linearly superposed to yield multicenter solutions. To show this, let us follow the reduction of the electrostatic sector of EMD to a threedimensional self-gravitating $\sigma$ model carried out in [25]. The dimensional reduction, achieved by

$$
d s^{2}=-f d t^{2}+f^{-1} \gamma_{i j} d x^{i} d x^{j}, \quad F_{i 0}=\frac{1}{\sqrt{2}} \partial_{i} v
$$

reduces the original 4-dimensional EMD equations to a three-dimensional problem deriving from the gravity coupled $\sigma$ model action

$$
S_{3}=\int d^{3} x \sqrt{\gamma}\left\{R_{\gamma}-G_{A B}(X) \partial_{i} X^{A} \partial_{j} X^{B} \gamma^{i j}\right\}
$$

where $R_{\gamma}$ is the Ricci scalar constructed from the 3-dimensional metric $\gamma_{i j}$, and $G_{A B}(X)$ is the target space metric

$$
d S^{2}=G_{A B} d X^{A} d X^{B}=\frac{d f^{2}}{2 f^{2}}-\frac{1}{f} \mathrm{e}^{-2 \alpha \phi} d v^{2}+2 d \phi^{2} .
$$

In the case where the potentials $f, v$ and $\phi$ depend on a single scalar potential $\sigma$, this potential can always [26, 27] be chosen to be harmonic $\left(\nabla_{\gamma}^{2} \sigma=0\right)$. 
The point $(f, v, \phi)$ then follows a geodesic in target space, null geodesics leading to a Ricci-flat, hence flat, reduced 3-space of metric $\gamma_{i j}$ [28, 29, 30]. The geodesics for the metric (3.3) are obtained by solving the system

$$
\begin{aligned}
f \ddot{f}-\dot{f}^{2} & =f \mathrm{e}^{-2 \alpha \phi} \dot{v}^{2}, \\
\left(f^{-1} e^{-2 \alpha \phi} \dot{v}\right) & =0, \\
2 f \ddot{\phi} & =\alpha \mathrm{e}^{-2 \alpha \phi} \dot{v}^{2} .
\end{aligned}
$$

It is straightforward to show that, for the choice $\gamma_{i j}=\delta_{i j}$, there are two kinds of null geodesics.

Null geodesics of the first, generic kind lead (up to a linear transformation on $\sigma)$ to the two-parameter $(c, k)$ family of singular solutions

$$
\begin{aligned}
f & =\left(\frac{c k^{\alpha^{2}} \mathrm{e}^{\alpha \sigma}}{\sin \sigma}\right)^{1+\gamma}, \\
v & =c \sqrt{1+\gamma} \cot \sigma, \\
\mathrm{e}^{2 \alpha \phi} & =\left(\frac{c}{k \sin \sigma}\right)^{1-\gamma} \mathrm{e}^{-(1+\gamma) \alpha \sigma} .
\end{aligned}
$$

For $\alpha=1$, these correspond to the electrostatic sector $(\chi=u=\kappa=0)$ of the type 2 (nondegenerate) solutions found in 30 (for instance, the solution (6.17) of [30] corresponds to $\left.c=1 / \sqrt{2}, k=\mathrm{e}^{-3 \pi / 4}\right)$. For $\alpha=\sqrt{3}$, the lift to five dimensions according to the Kaluza-Klein ansatz recalled in Sect. 5 leads to the metric

$d s_{5}^{2}=k \mathrm{e}^{\sigma / \sqrt{3}}\left(-c \sin \sigma d t^{2}+2 \cos \sigma d t d x^{5}+c^{-1} \sin \sigma\left(d x^{5}\right)^{2}\right)+k^{-2} \mathrm{e}^{-2 \sigma / \sqrt{3}} d \mathbf{x}^{2}$,

which belongs to the electrostatic sector of the class (a) of solutions found in 28 (Eq. (25)). Let us recall that the corresponding multi-center fivedimensional metric generated by the linear superposition

$$
\sigma=\sigma_{\infty}+\Sigma_{i}\left(c_{i} / r_{i}\right)
$$

is geodesically complete if all the $c_{i}$ are negative. Finally, for $\alpha=0$, the dilaton field does not decouple, so that the multicenter configurations (3.7) lead to the family of singular solutions of Einstein-Maxwell-massless scalar field theory:

$$
f=\frac{c^{2}}{\sin ^{2} \sigma}, \quad v=c \sqrt{2} \cot \sigma, \quad \phi=-\sigma .
$$


Null geodesics of the second, special kind lead (again up to a linear transformation on $\sigma$ ) to the one-parameter family of solutions

$$
\begin{aligned}
d s^{2} & =-\sigma^{-1-\gamma} d t^{2}+\sigma^{1+\gamma} d \mathbf{x}^{2}, \\
\mathcal{A} & =\nu \sqrt{\frac{1+\gamma}{2}} \sigma^{-1} d t, \quad e^{2 \alpha \phi}=\nu^{2} \sigma^{\gamma-1},
\end{aligned}
$$

where the harmonic function $\sigma$ may for instance be chosen in the multicenter form (3.11). As discussed in 31, for $\sigma_{\infty} \neq 0$ these are BPS (supersymmetric) asymptotically flat solutions generalizing the extreme $\left(r_{+}=r_{-}\right)$asymptotically flat black holes (2.3), while for $\sigma_{\infty}=0$ these multicenter solutions, which generalize (2.8)-(2.9) for $b=0$, are NAF. While the proof of supersymmetry given in 31 invokes asymptotic flatness, we conjecture that (as in the special case $\alpha=1$ [32, 2] ), these last NAF multicenter solutions are supersymmetric for all $\alpha$. By electro-magnetic duality one may derive from these electrostatic solutions the corresponding multicenter magnetostatic solutions.

\section{Quasilocal mass}

The mass of the static NAF self-gravitating configurations (2.8)-(2.9) was previously computed in [1]. For the sake of completeness, and to pave the way for a similar computation of the mass and spin of rotating NAF solutions in the next two sections, we outline this computation here. It employs the quasilocal energy approach developed among others by Brown and York [6] and put on a firm canonical basis by Hawking and Horowitz [7] (see also [8, 9] and references therein). Consider a spacetime region $M$ bounded by initial and final spacelike surfaces $\Sigma_{t_{1}}$ and $\Sigma_{t_{2}}$, and a timelike surface $\Sigma^{r}$ (not necessarily at spatial infinity), which we assume to be orthogonal to the $\Sigma_{t}$. In the canonical $1+3 \mathrm{ADM}$ decomposition [33, the metric and electromagnetic potential on $M$ are written as

$$
d s^{2}=-N^{2} d t^{2}+h_{i j}\left(d x^{i}+N^{i} d t\right)\left(d x^{j}+N^{j} d t\right), \quad \mathcal{A}=A_{0} d t+A_{i} d x^{i},
$$

where $h_{i j}$ is the induced metric on $\Sigma_{t}$. The three-surfaces $\Sigma_{t}$ and $\Sigma^{r}$ intersect on a two-surface $S_{t}^{r}$, with induced metric $\sigma_{\mu \nu}=h_{\mu \nu}-n_{\mu} n_{\nu}$, where $n^{i}$ is the unit normal to $\Sigma^{r}$. In the present case $S_{t}^{r}$ is a two-sphere of radius $r$, with

$$
\sigma_{a b} d x^{a} d x^{b}=r_{0}^{1+\gamma} r^{1-\gamma} d \Omega^{2}, \quad n^{r}=N=\sqrt{\frac{r^{\gamma}(r-b)}{r_{0}^{1+\gamma}}} .
$$


The action (2.1) in the region $M$, supplemented by boundary terms necessary to correctly account for Dirichlet boundary conditions on $\partial M$, can be rearranged after integration by parts as

$$
\begin{aligned}
S=\int d t & {\left[\int_{\Sigma_{t}}\left(p^{i j} \dot{h}_{i j}+p^{i} \dot{A}_{i}+p \dot{\phi}-N \mathcal{H}-N^{i} \mathcal{H}_{i}-A_{0} \mathcal{H}_{A}\right)-\right.} \\
& \left.-\oint_{S_{t}^{r}}\left(N \epsilon+2 N^{i} \pi_{i j} n^{j}+A_{0} \Pi^{r}\right)\right]
\end{aligned}
$$

where $p^{i j}, p^{i}$ and $p$ are the canonical momenta conjugate to $h_{i j}, A_{i}$ and $\phi$, and $\mathcal{H}, \mathcal{H}_{i}$, and $\mathcal{H}_{A}$ are the Hamiltonian, momentum and Coulomb constraints conjugate to the non-dynamical variables $N, N^{i}$ and $A_{0}$. These constraints vanish on shell, the Hamiltonian then reducing to the surface term

$$
H=\oint_{S_{t}^{r}}\left(N \epsilon+2 N^{i} \pi_{i j} n^{j}+A_{0} \Pi^{r}\right),
$$

where

$$
\epsilon=\frac{1}{8 \pi} k \sqrt{|\sigma|},
$$

$k$ being the trace of the extrinsic curvature of $S_{t}^{r}$ in $\Sigma_{t}$,

$$
k=-\sigma^{\mu \nu} D_{\mu} n_{\nu}
$$

(with $D_{\mu}$ the covariant derivative on $\Sigma_{t}$ ), the reduced momenta $\pi_{i j}=$ $(\sqrt{|\sigma|} / \sqrt{|h|}) p_{i j}$ are related to the extrinsic curvature of $\Sigma_{t}$,

$$
K_{i j}=-\frac{1}{2 N}\left(\dot{h}_{i j}-2 D_{(i} N_{j)}\right),
$$

by

$$
\pi_{i j}=\frac{1}{16 \pi} \sqrt{|\sigma|}\left(K h_{i j}-K_{i j}\right),
$$

and

$$
\Pi^{r}=\frac{1}{4 \pi} N \sqrt{|h|} \mathrm{e}^{-2 \alpha \phi} F^{r t} .
$$

The value of the Hamiltonian (4.4) generically diverges when the radius $r$ of the 2-sphere $S_{t}^{r}$ is taken to infinity. The quasilocal energy or mass is the difference between the value of this Hamiltonian and that for a suitable reference background or "vacuum" evaluated with the same boundary data 
for the fields $|\sigma|, N$ and $A_{0}$. Here, the area $4 \pi|\sigma|$ of a sphere of given radius $r$ depends only on the charge parameter $r_{0}$, hence the natural choice for the vacuum in a given charge sector ( $r_{0}$ fixed) is the extreme black hole solution with $b=0$. As the electric potential and dilaton field also depend only on $r_{0}$ (or vanish in the magnetic case), it follows that the electric contribution to the energy cancels between the black hole and background, leaving only the gravitostatic contribution given by the first term of (4.4) minus the corresponding background contribution. The computation of the extrinsic curvature of $S_{t}^{r}$ gives

$$
k=-(1-\gamma) \sqrt{\frac{r-b}{r_{0}^{1+\gamma} r^{2-\gamma}}},
$$

leading, after substraction, to the value for the mass

$$
\mathcal{M}=\frac{(1-\gamma) b}{4}
$$

quoted in Sect. 2.

Now we check agreement with the first law of black hole thermodynamics

$$
d \mathcal{M}=T d S+V_{h} d Q .
$$

The Hawking temperature $T$ is given by the surface gravity divided by $2 \pi$,

$$
T=\left.\frac{1}{2 \pi} n^{i} \partial_{i} N\right|_{h}=b^{\gamma} / 4 \pi r_{0}^{1+\gamma}
$$

while the black hole entropy is given by a quarter of the horizon area

$$
S=A_{h} / 4=\pi r_{0}^{1+\gamma} b^{1-\gamma} .
$$

A straightforward computation gives

$$
d \mathcal{M}-T d S=\frac{1+\gamma}{4} b \frac{d r_{0}}{r_{0}}
$$

In the magnetostatic case, the electric charge $Q$ is identically zero, so that the first law holds provided the scale parameter $r_{0}$ is held fixed during the variation. This makes sense, because we have defined the mass of a given black hole as the difference between its energy and the energy of the extreme black hole with the same value of $r_{0}$. 
In the electrostatic case, the electric charge being proportional to $r_{0} / \nu$, the first law again holds if the metric scale $r_{0}$ and dilaton scale $\nu$ are both held fixed. There is however another intriguing possibility. The electric potential associated with (2.9),

$$
V=-A_{0}=-\nu \sqrt{\frac{1+\gamma}{2}} \frac{(r-b)}{r_{0}}-C,
$$

is defined only up to an additive (gauge) constant $C$. In the case of, say, the Reissner-Nordström solution, the potential goes to a constant at spatial infinity, and there is a preferred gauge in which the potential vanishes at infinity and the first law (4.12) is valid. In the present case, the potential (4.16) diverges at spatial infinity, so that there is a priori no preferred gauge. It is however possible (just as in the case of charged black holes in $2+1$ gravity [34]) to choose the gauge $C$ such that the first law holds when both the parameters $b$ and $r_{0}$ are varied (with $\nu$ held fixed). Interestingly enough, with this choice

$$
C=\sqrt{\frac{1+\gamma}{2}} \frac{b \nu}{2 r_{0}},
$$

both the static versions of the differential first law (4.12) and of the Smarr formula

$$
\mathcal{M}=2 T S+V_{h} Q
$$

are satisfied. We suggest that a more careful thermodynamical treatment of these NAF charged black holes might explain the success of this apparently ad hoc procedure.

\section{Case $\alpha^{2}=3$ : rotating magnetic black holes}

Can these static non-asymptotically flat black holes be extended to rotating black holes? Such an extension was carried out in 2] for the special case $\alpha^{2}=1$, by taking advantage of the fact that EMD can be embedded in EMDA whose stationary sector can be reduced to a three-dimensional selfgravitating $S p(4, R) / U(2) \sigma$ model [35]. The strategy used was, first to find the $S p(4, R)$ group transformation $U$ generating the linear dilaton black holes of [2] from the Schwarzschild solution, then to apply the same transformation to the corresponding Kerr family of solutions to generate a family of rotating linear dilaton black holes. 
The symmetries of the target space of pure stationary EMD with general $\alpha$ were studied in [25], 36], where it was shown that this target space has a symmetric Riemannian space (or $\sigma$ model) structure only in the two cases $\alpha^{2}=0$ (Einstein-Maxwell theory, with the target space $S U(2,1) / S(U(1) \times U(2))$ and $\alpha^{2}=3$ (dimensionally reduced Kaluza-Klein theory with the target space $S L(3, R) / S O(3))$. In the first case, the static solutions (2.8) and (2.9) or (2.10) all correspond to Bertotti-Robinson spacetime viewed by different uniformly accelerating observers. As shown in [37, the rotating solutions generated from these by the $\sigma$ model procedure described above again correspond to the same Bertotti-Robinson spacetime viewed by uniformly rotating observers. Conversely, this means that the Kerr solution can be generated from the Schwarzschild solution by a combination of $S U(2,1)$ group transformations and uniform frame rotations, which can more generally be used to generate asymptotically dipole solutions of Einstein-Maxwell theory from asymptotically monopole solutions [38. We shall focus here on the other case $\alpha^{2}=3$, corresponding to $\gamma=-1 / 2$.

It is well-known that EMD theory with $\alpha^{2}=3$ is a dimensional reduction of 5-dimensional sourceless Kaluza-Klein theory, i.e. 5-dimensional vacuum Einstein gravity

$$
S=\frac{1}{16 \pi} \int d^{5} x \sqrt{\left|g_{5}\right|} R_{5}
$$

together with the assumption of a spacelike Killing vector $\partial / \partial x^{5}$. Indeed, making the standard Kaluza-Klein ansatz

$$
d s_{5}^{2}=e^{2 \phi / \sqrt{3}} d s_{4}^{2}+e^{-4 \phi / \sqrt{3}}\left(d x^{5}+2 A_{\mu} d x^{\mu}\right)^{2},
$$

and integrating out the cyclic coordinate $x^{5}$ reduces the action (5.1) to the EMD action (2.1) with $\alpha=\sqrt{3}$. It follows that the stationary solutions (i.e. solutions with a timelike Killing vector $\partial_{t}$ ) of $\alpha^{2}=3$ EMD are dimensional reductions of solutions of $5 \mathrm{D}$ vacuum gravity with two Killing vectors. As shown by Maison [39, this two-stationary sector of Kaluza-Klein theory may be reduced to a three-dimensional self-gravitating $S L(3, R) / S O(3) \sigma$ model. The metric ansatz appropriate for this five-to-three dimensional reduction is

$$
d s_{5}^{2}=\lambda_{a b}\left(d x^{a}+a_{i}^{a} d x^{i}\right)\left(d x^{b}+a_{j}^{b} d x^{j}\right)+\tau^{-1} \gamma_{i j} d x^{i} d x^{j},
$$

where $i=1, \ldots, 3, a=4,5\left(x^{4}=t\right), \tau=|\operatorname{det}(\lambda)|$, and the various fields depend only on the coordinates $x^{i}$. Using the 5 -dimensional Einstein equations, the magnetic-like vector potentials $a_{i}^{a}$ may be dualized to the scalar 
twist potentials $V_{a}$ according to

$$
V_{a, i} \equiv|\gamma|^{-1 / 2} \tau \lambda_{a b} \gamma_{i l} \epsilon^{j k l} a_{j, k}^{b}
$$

The remaining Einstein equations may then be written in the 3-dimensional self-gravitating $\sigma$-model form

$$
\left(\chi^{-1} \chi^{, i}\right)_{; i}=0, \quad R_{i j}=\frac{1}{4} \operatorname{Tr}\left(\chi^{-1} \chi_{, i} \chi^{-1} \chi_{, j}\right),
$$

where the 3 -metric is $\gamma_{i j}$, and $\chi$ is the unimodular symmetric $3 \times 3$ matrixvalued field

$$
\chi=\left(\begin{array}{cc}
\lambda_{a b}-\tau^{-1} V_{a} V_{b} & -\tau^{-1} V_{a} \\
-\tau^{-1} V_{b} & -\tau^{-1}
\end{array}\right)
$$

These equations are clearly invariant under $S L(3, R)$ transformations

$$
\chi \rightarrow U^{T} \chi U
$$

which therefore transform a stationary solution of $\left(\alpha^{2}=3\right)$ EMD into another solution with the same reduced 3-metric $\gamma_{i j}$.

We first consider the magnetic NAF black hole solution (2.8), (2.10) with $\alpha=\sqrt{3}$ (where we have chosen without loss of generality $\nu=1$ ), which leads according to (5.2) to the five-dimensional metric

$$
d s_{5}^{2}=-\frac{r-b}{r} d t^{2}+\frac{r}{r_{0}}\left(d x^{5}-r_{0} \cos \theta d \varphi\right)^{2}+\frac{r_{0}}{r-b}\left(d r^{2}+r(r-b) d \Omega^{2}\right) .
$$

The resulting representative matrix

$$
\chi_{m}=\left(\begin{array}{ccc}
-\frac{r-b}{r} & 0 & 0 \\
0 & -\frac{b r}{r_{0}(r-b)} & \frac{r}{r-b} \\
0 & \frac{r}{r-b} & -\frac{r_{0}}{r-b}
\end{array}\right)
$$

is a target space geodesic 28

$$
\chi=\eta \mathrm{e}^{A \sigma},
$$

with the harmonic potential

$$
\sigma=-\frac{r_{0}}{b} \ln \left|\frac{r-b}{r}\right|
$$


and the constant matrices $\eta$ and $A$

$$
\eta_{m}=\left(\begin{array}{ccc}
-1 & 0 & 0 \\
0 & -\frac{b}{r_{0}} & 1 \\
0 & 1 & 0
\end{array}\right), \quad A_{m}=\left(\begin{array}{ccc}
-\frac{b}{r_{0}} & 0 & 0 \\
0 & \frac{b}{r_{0}} & -1 \\
0 & 0 & 0
\end{array}\right)
$$

The metric (5.8) is regular on the axis $\sin \theta=0$ provided $x^{5}$ is periodic with period $4 \pi r_{0}$ 40. Making the coordinate transformation

$$
r=\frac{x^{2}}{4 r_{0}}, \quad x^{5}=r_{0} \eta,
$$

where $\eta$ is an angle, the metric (5.8) can be rearranged to

$$
d s_{5}^{2}=-\left(1-\frac{\mu}{x^{2}}\right) d t^{2}+\left(1-\frac{\mu}{x^{2}}\right)^{-1} d x^{2}+x^{2} d \Omega_{3}^{2},
$$

where

$$
d \Omega_{3}^{2}=\frac{1}{4}\left(d \theta^{2}+\sin ^{2} \theta d \varphi^{2}+(d \eta-\cos \theta d \varphi)^{2}\right)
$$

is the metric of the three-sphere. We recognize in (5.14) the static, spherically symmetric (in the four spatial dimensions) Tangherlini-Myers-Perry (TMP) five-dimensional black hole [14, 15] with mass parameter

$$
\mu=4 r_{0} b \text {. }
$$

So the static NAF magnetic black holes of $\left(\alpha^{2}=3\right)$ EMD are simply the dimensional reduction of the asymptotically flat TMP black holes relative to one of the azimuthal angles. Correspondingly, the NAF extreme $(b=0)$ magnetic black holes of $\left(\alpha^{2}=3\right)$ EMD are simply the dimensional reduction of five-dimensional Minkowski spacetime, their timelike singularity $r=0$ arising from the dimensional reduction of the (spurious if $x^{5}$ is periodic with period $4 \pi r_{0}$ ) Dirac string singularity of (5.8).

The reduced 3-metric $\gamma_{i j}$ of (5.8) coincides with that of the four-dimensional Schwarzschild solution with mass $M=b / 2$. It follows that there is an $S L(3, R)$ transformation $U_{S m}$ which transforms the five-dimensional trivially embedded Schwarzschild solution, i.e. the direct product of the fourdimensional Schwarzschild solution with the line (or the circle), with representative matrix

$$
\chi_{S}=\left(\begin{array}{ccc}
-\frac{r-b}{r} & 0 & 0 \\
0 & 1 & 0 \\
0 & 0 & -\frac{r}{r-b}
\end{array}\right)
$$


into the TMP black hole, written in the form (5.8), with representative $\chi_{m}$ :

$$
\chi_{m}=U_{m S} \chi_{S} U_{S m}, \quad U_{S m}=\left(\begin{array}{ccc}
1 & 0 & 0 \\
0 & 0 & \sqrt{\frac{r_{0}}{b}} \\
0 & -\sqrt{\frac{b}{r_{0}}} & \sqrt{\frac{r_{0}}{b}}
\end{array}\right)
$$

(with $U_{m S}=U_{S m}^{T}$ ).

As explained above, this transformation acting on the trivially embedded Kerr solution will generate rotating NAF magnetic black holes according to

$$
\chi_{m K}=U_{m S} \chi_{K} U_{S m} .
$$

The trivially embedded Kerr solution with mass $M_{0}=b / 2$ and rotation parameter $a_{0}$ is (in Boyer-Lindquist coordinates)

$$
\begin{aligned}
d s_{5}^{2}=-\left(\frac{\Gamma_{0}}{\Sigma_{0}}\right) & \left(d t-\omega_{0} d \varphi\right)^{2} \\
& +\Sigma_{0}\left(\frac{d r^{2}}{\Delta_{0}}+d \theta^{2}+\frac{\Delta_{0} \sin ^{2} \theta}{\Gamma_{0}} d \varphi^{2}\right)+\left(d x^{5}\right)^{2}
\end{aligned}
$$

with

$$
\begin{aligned}
& \Delta_{0}=r^{2}-b r+a_{0}^{2}, \quad \Sigma_{0}=r^{2}+a_{0}^{2} \cos ^{2} \theta \\
& \Gamma_{0}=\Delta_{0}-a_{0}^{2} \sin ^{2} \theta, \quad \omega_{0}=-\frac{a_{0} b r \sin ^{2} \theta}{\Gamma_{0}} .
\end{aligned}
$$

Acting on its representative matrix

$$
\chi_{K}=\frac{1}{\Gamma_{0}}\left(\begin{array}{ccc}
-(r-b)^{2}-a_{0}^{2} \cos ^{2} \theta & 0 & a_{0} b \cos \theta \\
0 & \Gamma_{0} & 0 \\
a_{0} b \cos \theta & 0 & -\Sigma_{0}
\end{array}\right)
$$

with the transformation (5.19), we obtain

$$
\chi_{m K}=\frac{1}{\Gamma_{0}}\left(\begin{array}{ccc}
-(r-b)^{2}-a_{0}^{2} \cos ^{2} \theta & -a_{0} b \sqrt{\frac{b}{r_{0}}} \cos \theta & a_{0} \sqrt{b r_{0}} \cos \theta \\
-a_{0} b \sqrt{\frac{b}{r_{0}}} \cos \theta & -\frac{b}{r_{0}} \Sigma_{0} & \Sigma_{0} \\
a_{0} \sqrt{b r_{0}} \cos \theta & \Sigma_{0} & -r_{0} r
\end{array}\right) .
$$

The resulting five-dimensional metric is

$$
\begin{aligned}
d s_{5}^{2}= & -\left(1-\frac{b}{r}\right) \psi^{2}+\frac{a_{0} \sqrt{b r_{0}} \cos \theta}{r} 2 \psi \xi+\frac{r_{0} \Sigma_{0}}{r} \xi^{2} \\
& +\frac{r_{0} r}{\Gamma_{0}}\left(\frac{\Gamma_{0}}{\Delta_{0}} d r^{2}+\Gamma_{0} d \theta^{2}+\Delta_{0} \sin ^{2} \theta d \varphi^{2}\right)
\end{aligned}
$$


with

$$
\psi=d t+\frac{a_{0} \sqrt{b r_{0}} r \sin ^{2} \theta}{\Gamma_{0}} d \varphi, \quad \xi=d \eta-\frac{\Delta_{0} \cos \theta}{\Gamma_{0}} d \varphi
$$

where as before we have put $x^{5}=r_{0} \eta$. Not surprisingly, this turns out to coincide with a subclass of rotating Myers-Perry black holes [15. The general rotating Myers-Perry (MP) black hole in five dimensions depends on two angular momentum parameters $a_{+}$and $a_{-}$and is given by

$$
\begin{aligned}
d s_{5}^{2}= & -d t^{2}+\frac{\mu}{\rho^{2}}\left[d t+a_{+} \sin ^{2} \bar{\theta} d \varphi_{+}+a_{-} \cos ^{2} \bar{\theta} d \varphi_{-}\right]^{2}+\rho^{2} d \bar{\theta}^{2} \\
& +\left(x^{2}+a_{+}^{2}\right) \sin ^{2} \bar{\theta} d \varphi_{+}^{2}+\left(x^{2}+a_{-}^{2}\right) \cos ^{2} \bar{\theta} d \varphi_{-}^{2}+\frac{\rho^{2} x^{2}}{\Theta} d x^{2}
\end{aligned}
$$

with

$$
\rho^{2}=x^{2}+a_{+}^{2} \cos ^{2} \bar{\theta}+a_{-}^{2} \sin ^{2} \bar{\theta}, \quad \Theta=\left(x^{2}+a_{+}^{2}\right)\left(x^{2}+a_{-}^{2}\right)-\mu x^{2} .
$$

After transforming the spatial coordinates to

$$
\bar{\theta}=\theta / 2, \quad \varphi_{ \pm}=(\varphi \pm \eta) / 2, \quad x^{2}=4 r_{0} r-\bar{a}^{2}
$$

the metric (5.25) is found to go over into the MP metric with two equal angular momentum parameters, the identification being

$$
\mu=4 r_{0} b, \quad a_{+}=a_{-}=\bar{a}=-\frac{4 r_{0} a_{0}}{\sqrt{\mu}} .
$$

The Kaluza-Klein dimensional reduction of (5.25) leads to the rotating NAF magnetic black hole solution

$$
\begin{aligned}
d s_{4}^{2}= & -\frac{\Gamma_{0}}{\sqrt{r_{0} r \Sigma_{0}}}\left(d t+\frac{\sqrt{b r_{0}} a_{0} r \sin ^{2} \theta}{\Gamma_{0}} d \varphi\right)^{2} \\
& +\sqrt{r_{0} r \Sigma_{0}}\left(\frac{d r^{2}}{\Delta_{0}}+d \theta^{2}+\frac{\Delta_{0} \sin ^{2} \theta}{\Gamma_{0}} d \varphi^{2}\right), \\
\mathcal{A}= & -\frac{r^{2}+a_{0}^{2}}{\Sigma_{0}} \frac{r_{0}}{2} \cos \theta\left(d \varphi-\frac{a_{0} \sqrt{b}}{\sqrt{r_{0}}\left(r^{2}+a_{0}^{2}\right)} d t\right), \\
\mathrm{e}^{2 \phi / \sqrt{3}=} & \sqrt{\frac{r_{0} r}{\Sigma_{0}}} .
\end{aligned}
$$


Similarly to the Kerr spacetime, the rotating spacetime (5.31) possesses two horizons at $r=r_{ \pm}$, with

$$
r_{ \pm}=\frac{1}{2}\left(b \pm \sqrt{b^{2}-4 a_{0}^{2}}\right)
$$

$r=r_{+}$corresponding to the event horizon. However, to the difference of the Kerr black hole, but similarly to the case of rotating linear dilaton black holes $(\alpha=1)$ [2], the metric (5.31) (as well as the five-dimensional metric (5.25) ) is singular on the timelike line $r=0$. Accordingly, the Penrose diagrams along the symmetry axis of these NAF rotating black holes are, for the three cases $b^{2}>4 a_{0}^{2}, b^{2}=4 a_{0}^{2}$ and $b^{2}<4 a_{0}^{2}$, identical to those of the Reissner-Nordström spacetime, with the charge replaced by the angular momentum parameter $a_{0}$. According to (5.32), this metric supports a rotating monopole magnetic field with magnetic charge

$$
\left.P=\frac{1}{4 \pi} \int F_{\theta \varphi} d \theta d \varphi=\frac{1}{2} A_{\varphi}\right]_{\theta=\pi}^{\theta=0}=-\frac{r_{0}}{2},
$$

as in the static case. The quasilocal computation of the mass $\mathcal{M}$ and angular momentum $J$ of these black holes, discussed in the next section, gives

$$
\mathcal{M}=\frac{3 b}{8}, \quad J=\frac{a_{0}}{2} \sqrt{b r_{0}} .
$$

In the massless case $M_{0}=b / 2=0$, we know that the Kerr spacetime reduces to Minkowski spacetime. Therefore the spatial part of the fivedimensional metric (5.20) is a (spheroidal coordinate) parametrization of flat three-space. This means that the solutions (5.31)-(5.33) with $b=0$ can be linearly superposed to give multicenter solutions similar to the Israel-WilsonPerjès 41] (IWP) solutions of Einstein-Maxwell theory. For $b=0$, the matrix $A_{m}$ of (5.12) is such that $A^{2}=0$, so that the target space geodesic (5.10) is null [28, 29]. The corresponding five-dimensional metric

$$
d s_{5}^{2}=-d t^{2}+d \mathbf{x}^{2}+\sigma^{-1}\left(d x^{5}+a_{i}^{5} d x^{i}\right)^{2},
$$

with

$$
\nabla \wedge a=\nabla \sigma
$$

reduces to the magnetic equivalent of the four-dimensional solution (3.13)(3.14) with $\alpha=\sqrt{3}(\gamma=-1 / 2)$. For the choice of the harmonic function

$$
\sigma=\frac{r_{0} r}{r^{2}+a_{0}^{2} \cos ^{2} \theta}
$$


this coincides with the singular solution (5.31)-(5.33) with $b=0$. As we have seen, for $a_{0}=0$ the corresponding five-dimensional metric reduces to five-dimensional Minkowski if $x^{5}$ is periodic with period $4 \pi r_{0}$. Accordingly, the static multi-center harmonic function (3.11) with $\sigma_{\infty}=0$ will lead to five-dimensional spacetimes with Dirac string singularities originating from the centers, unless all the residues $c_{i}$ are equal, in which case (5.37) will reduce again to five-dimensional Minkowski spacetime.

\section{Case $\alpha^{2}=3$ : dyonic black holes (magnetic sector)}

We have seen that the static NAF magnetic black holes of $\left(\alpha^{2}=3\right)$ EMD are a dimensional reduction of the five-dimensional TMP black holes, while their rotating generalisations (5.31)-(5.33) are a dimensional reduction of the MP black holes with two equal angular momentum parameters. One may surmise that the more general MP black holes (5.27) with two unequal angular momentum parameters should lead, by an appropriate dimensional reduction, to generalized four-dimensional NAF magnetic black holes with two extra quantum numbers, which as we will now show can be identified as four-dimensional angular momentum and electric charge associated with rotating dyonic black holes. Putting

$$
a_{ \pm}=\beta \pm \delta,
$$

transforming as before the spatial coordinates to

$$
\bar{\theta}=\theta / 2, \quad \varphi_{ \pm}=(\varphi \pm \eta) / 2, \quad x^{2}=4 r_{0} r-\left(\beta^{2}+\delta^{2}\right)
$$

carrying out the dimensional reduction (5.2) of (5.27) relative to $\partial_{5}=r_{0}^{-1} \partial_{\eta}$, and putting $\mu=4 r_{0} b$, we obtain

$$
\begin{aligned}
d s_{4}^{2}= & -\frac{\Gamma}{\sqrt{\Pi A}}(d t-\bar{\omega} d \varphi)^{2}+\sqrt{\Pi A}\left(\frac{d r^{2}}{\Delta}+d \theta^{2}+\frac{\Delta \sin ^{2} \theta}{\Gamma} d \varphi^{2}\right) \\
\mathcal{A}= & -\frac{r_{0}}{2 A}\left(\left[(\Delta+b r) \cos \theta-\frac{b \beta \delta}{2 r_{0}}-\frac{\beta \delta}{2 r_{0}}(r-b / 2) \sin ^{2} \theta\right] d \varphi\right. \\
& \left.\quad-\frac{b}{2 r_{0}}(\delta-\beta \cos \theta) d t\right) \\
\mathrm{e}^{2 \phi / \sqrt{3}}= & \sqrt{\frac{\Pi}{A}}
\end{aligned}
$$


with

$$
\begin{aligned}
& \Delta=(r-b / 2)^{2}-\frac{\bar{\beta}^{2} \bar{\delta}^{2}}{4 r_{0}^{2}}, \quad \Gamma=\Delta-\frac{\beta^{2} \bar{\delta}^{2}}{4 r_{0}^{2}} \sin ^{2} \theta \\
& A=r^{2}+\frac{b}{4 r_{0}}(\delta-\beta \cos \theta)^{2}-\frac{\beta^{2} \delta^{2}}{4 r_{0}^{2}} \cos ^{2} \theta, \quad \Pi=r_{0} r+\frac{\beta \delta}{2} \cos \theta,(6.6) \\
& \bar{\omega}=\frac{\beta b\left(r-\delta^{2} / 2 r_{0}\right)}{2 \Gamma} \sin ^{2} \theta \quad\left(\bar{\beta}^{2}=b r_{0}-\beta^{2}, \bar{\delta}^{2}=b r_{0}-\delta^{2}\right) .
\end{aligned}
$$

We see that the reduced spatial metric in (6.3) coincides with that of the Kerr metric with parameters $M^{2}=b \bar{\delta}^{2} / 4 r_{0}, a^{2}=\beta^{2} \bar{\delta}^{2} / 4 r_{0}^{2}$, meaning that the solutions (6.3)-(6.5) can also be generated from the trivially embedded Kerr metric, or from the rotating magnetic solutions (5.31)-(5.33), by a suitably chosen $S L(3, R)$ transformation.

The spacetimes (6.3) have two horizons $r=r_{ \pm}$, with

$$
r_{ \pm}=\frac{1}{2}\left(b \pm \frac{\bar{\beta} \bar{\delta}}{r_{0}}\right)
$$

if either $\beta^{2}<b r_{0}$ and $\delta^{2}<b r_{0}$, or $\beta^{2}>b r_{0}$ and $\delta^{2}>b r_{0}$. However, as discussed in [15], this second possibility does not lead to regular black holes. The values of $A_{\varphi}$ on the Dirac strings $\theta=0$ and $\theta=\pi$ are unchanged from their static $(\beta=0)$ values, so that the magnetic charge is again given by (5.35),

$$
P=-\frac{r_{0}}{2}
$$

In order to compute the electric charge, we evaluate

$$
\begin{aligned}
\Pi^{r} \equiv & \frac{1}{4 \pi} \sqrt{|g|} \mathrm{e}^{-2 \sqrt{3} \phi} F^{r t} \\
= & \frac{\sin \theta}{8 \pi} \frac{b r_{0}}{\Pi^{2}}\left((\delta-\beta \cos \theta) r^{2}-\frac{\beta \delta}{4 r_{0}}\left[\beta\left(1+\cos ^{2} \theta\right)-2 \delta \cos \theta\right] r\right. \\
& \left.\quad-\frac{\beta^{2} \delta^{3}}{8 r_{0}^{2}} \sin ^{2} \theta\right)
\end{aligned}
$$

leading after integration to

$$
Q=-\frac{b \delta}{2 r_{0}} .
$$


So the difference $\delta$ between the two angular momentum parameters of the original MP black hole (5.27) leads, after dimensional reduction, to a fourdimensional electric charge, while the sum $\beta$ leads to a four-dimensional angular momentum, which shall be computed below.

For $\delta=0\left(a_{-}=a_{+}\right)$, we recover the rotating magnetic black holes of the previous section, with

$$
a_{0}=-\sqrt{b / r_{0}} \beta / 2 .
$$

For $\beta=0\left(a_{-}=-a_{+}\right)$, the solution takes the form

$$
\begin{aligned}
d s_{4}^{2}= & -\frac{(r-b / 2)^{2}-b \bar{\delta}^{2} / 4 r_{0}}{\sqrt{r_{0} r\left(r^{2}+b \delta^{2} / 4 r_{0}\right)}} d t^{2} \\
& +\sqrt{r_{0} r\left(r^{2}+b \delta^{2} / 4 r_{0}\right)}\left(\frac{d r^{2}}{(r-b / 2)^{2}-b \bar{\delta}^{2} / 4 r_{0}}+d \Omega^{2}\right), \\
\mathcal{A}= & -\frac{r_{0}}{2} \cos \theta d \varphi+\frac{b \delta}{4\left(r^{2}+b \delta^{2} / 4 r_{0}\right)} d t \\
\mathrm{e}^{2 \phi / \sqrt{3}=} & \sqrt{\frac{r_{0} r}{r^{2}+b \delta^{2} / 4 r_{0}}},
\end{aligned}
$$

corresponding to static NAF dyonic black holes. Finally, for $b=0$ we recover the magnetostatic IWP solutions discussed at the end of the previous section.

The NAF black holes (6.3) become extreme for $\bar{\beta}=0\left(\beta^{2}=b r_{0}\right)$ or $\bar{\delta}=0$ $\left(\delta^{2}=b r_{0}\right)$, In this last case $(\bar{\delta}=0)$, it follows from (6.6) that $\Delta=\Gamma=$ $(r-b / 2)^{2}$, so that the solutions (6.3) have conformally flat spatial sections. Again, this means that these solutions can be linearly superposed to give multicenter solutions. We only discuss here the static case $\left(\beta=0, \delta^{2}=b r_{0}\right.$, i.e. $a_{+}=-a_{-}=\sqrt{\mu / 4}$ ). In this case the solution (6.12)-(6.14) is of the form (5.10), with the matrix $\eta$ given in (5.12),

$$
A=\left(\begin{array}{ccc}
-1 & -\sqrt{\frac{b}{r_{0}}} & 0 \\
0 & 1 & -\frac{r_{0}}{b} \\
\sqrt{\frac{b}{r_{0}}} & \frac{b}{r_{0}} & 0
\end{array}\right),
$$

and $\sigma=b /(r-b / 2)$. This matrix $A$ is such that $A^{2} \neq 0, A^{3}=0$. The corresponding multicenter solution thus belongs to the class (b) discussed in [28] and is given by

$$
d s_{4}^{2}=-f d t^{2}+f^{-1} d \mathbf{x}^{2}, \quad f=\sqrt{\frac{b}{r_{0}}} \sqrt{\sigma(1+\sigma / 2)\left(1+\sigma+\sigma^{2} / 2\right)}(6.16)
$$




$$
\begin{aligned}
\mathcal{A} & =\frac{r_{0}}{2 b} a_{i} d x^{i}+\sqrt{\frac{r_{0}}{b}} \frac{\sigma^{2}}{4\left(1+\sigma+\sigma^{2} / 2\right)} d t \\
\mathrm{e}^{2 \phi / \sqrt{3}} & =\sqrt{\frac{r_{0}}{b}} \sqrt{\frac{\sigma(1+\sigma / 2)}{1+\sigma+\sigma^{2} / 2}},
\end{aligned}
$$

with

$$
\sigma=\Sigma_{i}\left(c_{i} / r_{i}\right), \quad \nabla \wedge a=\nabla \sigma .
$$

Now we compute the mass and angular momentum of the four-dimensional $\alpha=\sqrt{3}$ rotating dyonic NAF black holes. The mass is again given by the limit $r \rightarrow \infty$ of the difference between the values of the quasilocal Hamiltonian (4.4) for the rotating $\left(r_{0}, b, \beta, \delta\right)$ black hole and for the $\left(r_{0}, 0,0,0\right)$ background. The ADM form of the metric (6.3) is

$$
\begin{aligned}
d s^{2}= & -\frac{\sqrt{\Pi A} \Delta}{\Omega} d t^{2}+\sqrt{\Pi A}\left[\frac{d r^{2}}{\Delta}+d \theta^{2}\right. \\
& \left.+\frac{\Omega}{\Pi A} \sin ^{2} \theta\left(d \varphi+\frac{\beta b\left(r-\delta^{2} / 2 r_{0}\right)}{2 \Omega} d t\right)^{2}\right]
\end{aligned}
$$

with

$$
\Omega=\Pi \Delta+b r_{0}\left(r^{2}-\beta^{2} \delta^{2} / 4 r_{0}^{2}\right) .
$$

Keeping only the leading asymptotic $(r \rightarrow \infty)$ monopole and dipole contributions, we obtain

$$
\sigma_{a b} d x^{a} d x^{b} \simeq r \sqrt{\Pi} d \Omega^{2}, \quad n^{r} \simeq N \simeq \frac{\sqrt{r-b}}{\Pi^{1 / 4}}, \quad N^{\varphi} \simeq \frac{\beta b}{2 r_{0} r^{2}}
$$

leading to the asymptotic extrinsic curvature

$$
k \simeq-\frac{3}{2} r_{0}^{-1 / 4} r^{-3 / 4}\left(1-\frac{b}{2 r}-\frac{7}{24} \frac{\beta \delta}{r_{0} r} \cos \theta\right) .
$$

So the value of the first term $N \epsilon$ of the integrand of (4.4) differs from the magnetostatic $(\beta=\delta=0)$ value only by a term in $\sin \theta \cos \theta$ which disappears after integration over the sphere. The second term of the integrand behaves asymptotically as the product of $N^{\varphi}$, in $r^{-2}$, with

$$
\pi_{\varphi r} n^{r} \simeq \frac{b \beta}{32 \pi} \sin ^{3} \theta
$$


so that its contribution to the quasilocal mass vanishes when the radius of the sphere $S_{r}^{t}$ is taken to infinity. Finally, the third term of the integrand behaves asymptotically as the product of

$$
A_{0} \simeq \frac{b}{4 r_{0} r^{2}}(\delta-\beta \cos \theta)
$$

with

$$
\Pi^{r} \simeq \frac{b \sin \theta}{8 \pi r_{0}}(\delta-\beta \cos \theta),
$$

and again goes to zero when $r \rightarrow \infty$. It follows that the mass of the rotating dyonic black hole is unchanged from its magnetostatic value,

$$
\mathcal{M}=\frac{3 b}{8} .
$$

The quasilocal momenta are obtained from the Hamiltonian by carrying out an infinitesimal gauge transformation $\delta x^{i}=\delta \xi^{i} t$ and evaluating the response $P_{i}=\delta H / \delta \xi^{i}$. For the angular momentum $J=P_{\varphi}$, one obtains [43]

$$
J=-\oint_{S_{t}^{r}}\left(2 \pi_{\varphi r} n^{r}+A_{\varphi} \Pi^{r}\right)
$$

(the static background does not contribute). From the preceding evaluations and

$$
A_{\varphi} \simeq-\frac{r_{0}}{2} \cos \theta,
$$

one obtains asymptotically

$$
A_{\varphi} \Pi^{r} \simeq-\frac{b \sin \theta}{16 \pi}\left(\delta \cos \theta-\beta \cos ^{2} \theta\right) .
$$

The first term disappears after integration over the sphere, while the second term combines with the gravitational contribution (6.24) to yield

$$
J=-\frac{b \beta}{4} .
$$

It is remarkable that, as in the case of the linear dilaton black holes [2], the (often ignored) electromagnetic contribution to the angular momentum is of the same order as the purely gravitational contribution.

There is a simple relation between these values of the mass and angular momentum for the four-dimensional NAF black holes (6.3)-(6.5) and the 
values of the corresponding quantities [15] for the five-dimensional MyersPerry black holes (5.27)

$$
M_{5}=\frac{3 \pi}{8} \mu, \quad J_{5 \pm}=\frac{2}{3} M_{5} a_{ \pm},
$$

which may be understood in terms of the dimensional reduction procedure. This amounts to integrating out the cyclic coordinate $x^{5}=r_{0} \eta$ from the fivedimensional action. The perimeter $\oint d x^{5}$ may be evaluated by computing the area $A_{3}\left(=2 \pi^{2}\right)$ of the three-sphere from (5.15),

$$
A_{3}=\left(\frac{1}{4}\right)^{3 / 2} A_{2} \oint d \eta
$$

Using the relations (5.16) and (6.1), we then check that the ratio between the four- and five-dimensional parameters is equal to the perimeter of the cyclic dimension:

$$
\frac{M_{5}}{M_{4}}=-\frac{J_{5+}+J_{5-}}{2 J_{4}}=\frac{J_{5+}-J_{5-}}{2 Q P}=\oint d x^{5}=4 \pi r_{0},
$$

We now check that these values of the black hole mass and angular momentum, together with the other physical parameters of the rotating black holes (6.3)-(6.5), satisfy the generalized first law of thermodynamics for dyonic, rotating black holes [13,

$$
d \mathcal{M}=T d S+\Omega_{h} d J+V_{h} d Q+W_{h} d P .
$$

In (6.35), the Hawking temperature and entropy are

$$
T=\frac{\bar{\beta} \bar{\delta}}{2 \pi b r_{0}(\bar{\beta}+\bar{\delta})}, \quad S=\frac{\pi b}{2}(\bar{\beta}+\bar{\delta}) .
$$

$\Omega_{h}$ is the horizon angular velocity

$$
\Omega_{h}=-\left.N^{\varphi}\right|_{h}=-\frac{\beta \bar{\delta}}{b r_{0}(\bar{\beta}+\bar{\delta})} .
$$

$V_{h}$ is the proper horizon electric potential, i.e. the electric potential in the horizon rest frame ${ }^{2}$, evaluated on the horizon $r=r_{+}$,

$$
V_{h}=-\left(A_{0}-N^{\varphi} A_{\varphi}\right)_{h}=-\frac{\delta \bar{\beta}}{2 b(\bar{\beta}+\bar{\delta})},
$$

\footnotetext{
${ }^{2}$ The electric potential in (6.4) is uniquely defined by the condition that it vanish at spatial infinity.
} 
while $W_{h}$ is the proper horizon magnetic potential, which (in the absence of a better definition) we shall define by duality as the proper horizon electric potential of the dyonic black hole of the electric type (see Section 7) with electric charge $P$, magnetic charge $Q$, and angular momentum $-J$ [13], given in (7.37). A straightforward computation then shows that when the three parameters $b, \bar{\beta}, \bar{\delta}$ are varied independently, the generalized first law (6.35) is satisfied identically, provided the scale parameter $r_{0}$ is held fixed.

One also easily checks that the rotating dyonic black holes satisfy the Smarr-like formula

$$
\mathcal{M}=\frac{3}{4}\left(2 T S+2 \Omega_{h} J+V_{h} Q+W_{h} P\right),
$$

similar to the Smarr formula for the asymptotically flat dyonic black holes of Kaluza-Klein theory [13], except for the anomalous factor 3/4. This factor may be understood by comparing (6.39) (where $V_{h} Q+W_{h} P=2 V_{h} Q$ on account of (7.38) ) with the 5-dimensional Myers-Perry Smarr formula [15]

$$
M_{5}=\frac{3}{4}\left(2 T S_{5}+2 \Omega_{+} J_{+}+2 \Omega_{-} J_{-}\right),
$$

where the 5-dimensional entropy (the quarter of the 5-dimensional horizon area) is $S_{5}=4 \pi r_{0} S_{4}$, and $\Omega_{ \pm}$are the two horizon angular velocities.

\section{Case $\alpha^{2}=3$ : dyonic black holes (electric sector)}

From these NAF rotating dyonic black holes of the magnetic type, one can construct a dual sector of NAF rotating dyonic black holes of the electric type by the electromagnetic duality transformation (2.2). Before doing this, it is instructive to first consider the purely electrostatic NAF black holes and their rotating counterparts. For $\alpha=\sqrt{3}$, we obtain for our electric NAF black hole solution (2.8)-(2.9)

$$
\mathrm{e}^{2 \phi / \sqrt{3}}=\left(\frac{r}{r_{0}}\right)^{1 / 2}, \quad A_{0}=\frac{r-b}{2 r_{0}},
$$

leading for the 5-dimensional metric (5.2) to

$$
d s_{5}^{2}=-\frac{r-b}{r_{0}} d t^{2}+\frac{r_{0}}{r}\left(d x^{5}+\frac{r-b}{r_{0}} d t\right)^{2}+\frac{r}{r-b}\left(d r^{2}+r(r-b) d \Omega^{2}\right) .
$$


The corresponding representative matrix $\chi_{e}$

$$
\chi_{e}=\left(\begin{array}{ccc}
-\frac{b(r-b)}{r_{0} r} & \frac{r-b}{r} & 0 \\
\frac{r-b}{r} & \frac{r_{0}}{r} & 0 \\
0 & 0 & -\frac{r}{r-b}
\end{array}\right)
$$

is of the form (5.10) with the harmonic potential (5.11) and the constant matrices

$$
\eta_{e}=\left(\begin{array}{ccc}
-\frac{b}{r_{0}} & 1 & 0 \\
1 & 0 & 0 \\
0 & 0 & -1
\end{array}\right), \quad A_{e}=\left(\begin{array}{ccc}
-\frac{b}{r_{0}} & 1 & 0 \\
0 & 0 & 0 \\
0 & 0 & \frac{b}{r_{0}}
\end{array}\right) .
$$

Again, the reduced 3-metric $\gamma_{i j}$ in (17.2) coincides with that of the Schwarzschild solution with mass $M=b / 2$, meaning that the matrix $\chi_{e}$ is an $S L(3, R)$ transform of the matrix (5.17) for the trivially embedded Schwarzschild solution,

$$
\chi_{e}=U_{e S} \chi_{S} U_{S e}, \quad U_{S e}=\left(\begin{array}{ccc}
\sqrt{\frac{b}{r_{0}}} & -\sqrt{\frac{r_{0}}{b}} & 0 \\
0 & \sqrt{\frac{r_{0}}{b}} & 0 \\
0 & 0 & 1
\end{array}\right) .
$$

Equivalently, one can simply note that the 5-dimensional metric (7.2) can be rearranged as

$$
d s_{5}^{2}=-\frac{b}{r_{0}}\left(1-\frac{b}{r}\right)\left(d t-\frac{r_{0}}{b} d x^{5}\right)^{2}+\frac{r}{r-b} d r^{2}+r^{2} d \Omega^{2}+\frac{r_{0}}{b}\left(d x^{5}\right)^{2},
$$

which is clearly the product of the Schwarzschild solution for an observer with time

$$
t^{\prime}=\sqrt{\frac{b}{r_{0}}}\left(t-\frac{r_{0}}{b} x^{5}\right)
$$

with the line or the circle. So the NAF electric black hole solution (2.8)(2.9) with $\alpha=\sqrt{3}$ is simply a twisted dimensional reduction of the trivial 5-dimensional embedding of the Schwarzschild solution with respect to the Killing vector

$$
\partial_{5}^{\prime}=\sqrt{\frac{r_{0}}{b}}\left(\partial_{5}-\partial_{t}\right)
$$

Note that the "twist" 3 in the relation (7.7) between the two times is not innocent in the traditional Kaluza-Klein interpretation, where the fifth coordinate $x^{5}$ varies on the so-called Klein circle so that, assuming the domain of

\footnotetext{
${ }^{3}$ This term is used here in a different sense from that used in (5.4).
} 
the time $t$ to be the real axis, the corresponding Schwarzschild time $t^{\prime}$ must be periodically identified ${ }^{4}$.

Let us recall that the well-known asymptotically flat electrically charged Kaluza-Klein black holes, given by (2.3) with $\alpha=\sqrt{3}$, may also be obtained from the trivially embedded Schwarzschild solution by a twisted dimensional reduction, this time with respect to the boosted Killing vector

$$
\partial_{5}^{\prime \prime}=\cosh \xi \partial_{5}+\sinh \xi \partial_{t}
$$

(the $S L(3, R)$ transformations preserving staticity and asymptotic flatness belong to the subgroup $S O(1,1)$ of Lorentz boosts in the 2-space $\left(t, x^{5}\right)$ ). The product of this boost with the transformation (7.8) - an infinite boost together with an unessential rescaling - being again a rescaled infinite boost, it follows that the twisted dimensional reduction with respect to (7.8) of the AF electrostatic Kaluza-Klein black holes will also lead to essentially the same (up to a rescaling of $r_{0}$ ) NAF electrostatic black hole ${ }^{5}$.

We are now in a position to generate the rotating electric NAF black hole solutions of $\left(\alpha^{2}=3\right)$ EMD. We have noted that the transformation from the Schwarzschild solution of $\left(\alpha^{2}=3\right)$ EMD to the static solution (2.8)-(2.9) boils down in this case to the "twist in time" (17.7). Therefore the rotating solutions are simply the twisted dimensional reduction of the trivially embedded Kerr solutions with mass $b / 2$ and rotation parameter $a_{0}$, i.e. their 5-dimensional metric is (in Boyer-Lindquist coordinates)

$$
\begin{aligned}
d s_{5}^{2}= & -\frac{b}{r_{0}}\left(\frac{\Gamma_{0}}{\Sigma_{0}}\right)\left(d t-\sqrt{\frac{r_{0}}{b}} \omega_{0} d \varphi-\frac{r_{0}}{b} d x^{5}\right)^{2} \\
& +\Sigma_{0}\left(\frac{d r^{2}}{\Delta_{0}}+d \theta^{2}+\frac{\Delta_{0} \sin ^{2} \theta}{\Gamma_{0}} d \varphi^{2}\right)+\frac{r_{0}}{b}\left(d x^{5}\right)^{2}
\end{aligned}
$$

Reversing the steps which led from the static solutions (2.8) $-(2.9)$ with $\alpha=$ $\sqrt{3}$ to (7.6), we obtain the rotating NAF electric black holes of $\left(\alpha^{2}=3\right)$ EMD

$$
d s_{4}^{2}=-\frac{\Gamma_{0}}{\sqrt{r_{0} r \Sigma_{0}}}\left(d t+\frac{\sqrt{b r_{0}} a_{0} r \sin ^{2} \theta}{\Gamma_{0}} d \varphi\right)^{2}
$$

\footnotetext{
${ }^{4} \mathrm{~A}$ similar phenomenon occurs for the spinning point particle solution of $2+1$ gravity 42] which is obtained from the non-spinning solution $d s_{3}^{2}=-d t^{2}+d r^{2}+\alpha^{2} r^{2} d \theta^{2}$ by the replacement $t \rightarrow t+\omega \theta$ ( $\alpha, \omega$ constant).

${ }^{5}$ In this case there is no simple relation between the electric charge of the AF black hole and that of the NAF black hole.
} 


$$
\begin{aligned}
& +\sqrt{r_{0} r \Sigma_{0}}\left(\frac{d r^{2}}{\Delta_{0}}+d \theta^{2}+\frac{\Delta_{0} \sin ^{2} \theta}{\Gamma_{0}} d \varphi^{2}\right), \\
\mathcal{A}= & \frac{1}{2 r_{0} r}\left(\Gamma_{0} d t+\sqrt{b r_{0}} a_{0} r \sin ^{2} \theta d \varphi\right), \\
\mathrm{e}^{2 \phi / \sqrt{3}}= & \sqrt{\frac{\Sigma_{0}}{r_{0} r}} .
\end{aligned}
$$

The corresponding electric field

$$
F=\frac{r^{2}-a_{0}^{2} \cos ^{2} \theta}{2 r_{0} r^{2}} d r \wedge d t+\sqrt{\frac{b}{r_{0}}} a_{0} \cos \theta \sin \theta d \theta \wedge\left(d \varphi-\frac{a_{0}}{\sqrt{b r_{0}} r} d t\right)
$$

leads again to the electric charge (2.11),

$$
Q=\frac{r_{0}}{2}
$$

As in the magnetic case, for $b=0$ the reduced three-dimensional metric in (17.11) is flat, so that the solution (7.11) $-(7.13)$ with $b=0$ can be generalized to multicenter solutions. Using the null geodesic construction with (7.4) for $b=0$ as input, it is easy to show that the corresponding 5 -dimensional metric is 44

$$
d s_{5}^{2}=2 d t d x^{5}+\sigma\left(d x^{5}\right)^{2}+d \mathbf{x}^{2}
$$

in accordance with (3.13)-(3.14) for $\alpha=\sqrt{3}$. The solution (7.11)-(7.13) with $b=0$ is recovered for the choice (5.39) of the harmonic function $\sigma$.

Clearly, the rotating electric solutions (7.11)-(7.13) are dual to the rotating magnetic solutions (5.31)-(5.33). More generally, acting on the rotating dyonic solutions of the magnetic type (6.3)-(6.5) with the duality transformation (2.2) with the lower sign, we obtain the new sector of NAF rotating dyonic black hole solutions:

$$
\begin{aligned}
d s_{4}^{2}= & -\frac{\Gamma}{\sqrt{\Pi A}}(d t-\bar{\omega} d \varphi)^{2}+\sqrt{\Pi A}\left(\frac{d r^{2}}{\Delta}+d \theta^{2}+\frac{\Delta \sin ^{2} \theta}{\Gamma} d \varphi^{2}\right) 7, . \\
\mathcal{A}= & \frac{1}{2 \Pi}\left(\left[A-b\left(r+\delta^{2} / 2 r_{0}\right)\right] d t-\frac{b}{r_{0}}[\delta \Pi \cos \theta\right. \\
& \left.\left.\quad+\frac{\beta r_{0}}{2}\left(r+\delta^{2} / 2 r_{0}\right) \sin ^{2} \theta\right] d \varphi\right) \\
\mathrm{e}^{2 \phi / \sqrt{3}}= & \sqrt{\frac{A}{\Pi}}
\end{aligned}
$$


(the functions $\Delta, \Gamma, A, \Pi, \bar{\omega}$ are given in (6.6)) with electric and magnetic charges

$$
Q=\frac{r_{0}}{2}, \quad P=-\frac{b \delta}{2 r_{0}} .
$$

Lifting these solutions to five dimensions according to (5.2), and carrying out the inverse "twist" transformation

$$
t=\sqrt{\frac{r_{0}}{b}}\left(t^{\prime}+x^{\prime 5}\right), \quad x^{5}=\sqrt{\frac{b}{r_{0}}} x^{\prime 5},
$$

we arrive after some calculations to the asymptotically flat five-dimensional metric

$$
\begin{gathered}
d s_{5}^{2}=-\frac{\Gamma}{B}\left(d t^{\prime}-\omega d \varphi\right)^{2}+A\left(\frac{d r^{2}}{\Delta}+d \theta^{2}+\frac{\Delta \sin ^{2} \theta}{\Gamma} d \varphi^{2}\right) \\
+\frac{B}{A}\left(d x^{\prime 5}+2 \hat{A}_{\mu} d x^{\prime \mu}\right)^{2}
\end{gathered}
$$

with

$$
\begin{aligned}
B & =\left(r-\frac{\delta^{2}}{2 r_{0}}\right)^{2}+\frac{\bar{\delta}^{2}}{4 r_{0}^{2}}(\delta-\beta \cos \theta)^{2} \\
\omega & =-\sqrt{\frac{b}{r_{0}}} \frac{\delta \Delta \cos \theta-\left(\beta \bar{\delta}^{2} / 2 r_{0}\right) r \sin ^{2} \theta}{\Gamma} \\
\hat{A}_{0} & =-\frac{\delta}{2 r_{0}} \frac{\delta(r-b / 2)+\left(\beta \bar{\delta}^{2} / 2 r_{0}\right) \cos \theta}{B} \\
\hat{A}_{\varphi} & =-\frac{\delta}{2} \sqrt{\frac{b}{r_{0}}} \frac{\left[r^{2}-\left(b r_{0} \delta^{2}-\beta^{2} \bar{\delta}^{2}\right) / 4 r_{0}^{2}\right] \cos \theta+\left(\beta \delta / 2 r_{0}\right)(r-b / 2) \sin ^{2} \theta}{B} .(7.25)
\end{aligned}
$$

The asymptotically flat metric (17.22) describes rotating dyonic black holes with NUT charge, generalizing the five-dimensional rotating dyonic black holes given by Rasheed [13. The corresponding mass, scalar charge, NUT charge, electric charge, magnetic charge, and angular momentum are given by

$$
\begin{aligned}
M_{A F} & =\frac{b}{2}-\frac{\delta^{2}}{4 r_{0}}, \quad \Sigma_{A F}=-\frac{\delta^{2} \sqrt{3}}{4 r_{0}}, \quad N_{A F}=\frac{\delta}{2} \sqrt{\frac{b}{r_{0}}} \\
Q_{A F} & =-\frac{\delta^{2}}{2 r_{0}}, \quad P_{A F}=-\frac{\delta}{2} \sqrt{\frac{b}{r_{0}}}, \\
J_{A F} & =\frac{\beta \bar{\delta}^{2}}{4 r_{0}} \sqrt{\frac{b}{r_{0}}} .
\end{aligned}
$$


There is no simple relation between these and the corresponding charges for the NAF dyonic black hole solutions (17.17)-(7.19). The appearance here of an AF NUT charge equal in magnitude to the AF magnetic charge is easy to understand. Generically, the twist transformation (7.8) acting on a magnetized AF solution will generate a NUTted NAF solution, the NAF NUT charge vanishing only if $N_{A F}+P_{A F}=0$. Recalling that the combination of any $S O(1,1)$ boost (7.9) with the twist acting on an AF Kaluza-Klein black hole will lead to essentially the same $\xi=0$ NAF black hole solution, we conclude that that the twist transformation acting on any NUTted dyonic AF black hole with $N_{A F}+P_{A F}=0$ will generate a NUTless dyonic NAF black hole of the electric type (7.17)-(7.19), which as we have seen is equivalent by electromagnetic duality to the dimensional reduction of a Myers-Perry black hole (5.27) relative to $\partial_{\eta}=\partial_{\varphi_{+}}-\partial_{\varphi_{-}}$. More generally, we conjecture that the twist transformation acting on a generic NUTted dyonic AF black hole will generate a NUTted dyonic NAF black hole, equivalent by electromagnetic duality to the dimensional reduction of a twisted Myers-Perry black hole (5.27). This conjecture is proven in the Appendix in the case where the original five-dimensional solution is the trivially embedded Taub-NUT solution.

Again, as in the magnetic case, for $\bar{\delta}=0\left(\delta^{2}=b r_{0}\right)$ these dyonic black hole solutions become extreme and can be linearly superposed to give multicenter IWP solutions. Remarkably, the twisted asymptotically flat metric (7.22) reduces in this case to the static, pure NUT form

$$
\begin{aligned}
d s_{5}^{2}=- & \left(d t^{\prime}+b \cos \theta d \varphi\right)^{2}+\frac{A}{(r-b / 2)^{2}}\left(d r^{2}+(r-b / 2)^{2} d \Omega^{2}\right) \\
+ & \frac{(r-b / 2)^{2}}{A}\left(d x^{\prime 5}+2 \hat{A}_{\mu} d x^{\prime \mu}\right)^{2}
\end{aligned}
$$

with $J_{A F}=0$, so that the parameter $\beta$ seems to be irrelevant. In the case $\beta=0$, the solution (7.28) is of the form (5.10) with $\eta=\operatorname{diag}(-1,1,-1)$,

$$
A=\left(\begin{array}{rrr}
0 & 1 & 1 \\
-1 & -1 & -1 \\
1 & 1 & 1
\end{array}\right)
$$

and $\sigma=b /(r-b / 2)$. Again, this matrix $A$ is such that $A^{2} \neq 0, A^{3}=0$. After carrying out the twist transformation, we arrive at the NAF multi-extreme 
black hole solution given by (6.16) and

$$
\begin{aligned}
\mathcal{A} & =\frac{b}{2 r_{0}} \frac{1-\sigma^{2} / 2}{\sigma(1+\sigma / 2)} d t+\frac{1}{2} \sqrt{\frac{b}{r_{0}}} a_{i} d x^{i}, \\
\mathrm{e}^{2 \phi / \sqrt{3}} & =\sqrt{\frac{b}{r_{0}}} \sqrt{\frac{1+\sigma+\sigma^{2} / 2}{\sigma(1+\sigma / 2)}},
\end{aligned}
$$

where $\sigma$ and $a$ are given by (6.19).

Let us now compute the quasilocal mass and angular momentum of the "electric" dyonic black holes (7.17)-(7.19). The spacetime metric is the same as for the "magnetic" dyonic black holes, so that the gravitational contributions to the quasilocal energy and angular momentum are the same. To compute the electromagnetic contributions, we evaluate the asymptotic behavior of the electric field density

$$
\begin{aligned}
\Pi_{(e)}^{r} & \equiv \frac{1}{4 \pi} \sqrt{|g|} \mathrm{e}^{-2 \sqrt{3} \phi} F^{r t}=-\frac{1}{4 \pi} F_{(m) \theta \varphi} \\
& \simeq-\frac{1}{8 \pi}\left(r_{0} \sin \theta+\beta \delta \sin \theta \cos \theta\right),
\end{aligned}
$$

where $F_{m}$ is the dual electromagnetic field derived from (6.4). The electromagnetic contribution to the quasilocal mass is the integral over a large sphere of the product of

$$
A_{0} \simeq \frac{r}{2 r_{0}}
$$

(a fixed boundary data) with the substracted electric field density

$$
\Pi^{r}-\left.\Pi^{r}\right|_{0} \simeq-\frac{\beta \delta}{8 \pi r} \sin \theta \cos \theta
$$

(where the index 0 stands for the static background $(b=\beta=\delta=0)$ ). This product goes to the finite value $-\left(\beta \delta / 16 \pi r_{0}\right) \sin \theta \cos \theta$, which however averages to zero after integration over the sphere, so that the mass is the same as in the magnetic case. The electromagnetic contribution to the quasilocal angular momentum is the integral of

$$
A_{\varphi} \Pi^{r} \simeq \frac{b \sin \theta}{16 \pi}\left(\delta \cos \theta+\beta \sin ^{2} \theta\right)
$$

(compare with (6.30). Again the first term disappears after averaging over the sphere, while the integral of the second term is the same as in the magnetic case. So the mass and angular momentum of these "electric" dyonic 
black holes are, not surprisingly, the same as those of their dual magnetic counterparts

$$
\mathcal{M}=\frac{3 b}{8}, \quad J=-\frac{b \beta}{4} .
$$

Finally we return to the question of the validity of the generalized first law (6.35) for rotating dyonic black holes. For a black hole of the magnetic sector (index $(m)$ ), the potential $W_{(m) h}$ is the proper horizon electric potential for the dyonic black hole of the electric sector (index $(e)$ ) with electric and magnetic charges exchanged, and opposite angular momentum, i.e. (compare (6.10), (6.8), (6.31) on the one hand and (7.20), (77.36) on the other hand) the charge and parity conjugate $\left(A_{0} \rightarrow-A_{0}, N^{\varphi} \rightarrow-N^{\varphi}\right)$ of the dyonic black hole (17.17)-(7.19) . We obtain for this quantity the value

$$
W_{(m) h}=-V_{(e) h}=\left(A_{(e) 0}-N_{(e)}^{\varphi} A_{(e) \varphi}\right)_{h}=-\frac{\delta^{2} \bar{\beta}}{2 r_{0}^{2}(\bar{\beta}+\bar{\delta})},
$$

which was used in Sect. 6 to check the generalized first law (6.35) in the magnetic sector. Symmetrically, it follows from this definition of the proper horizon magnetic potential that $W_{(e) h}=V_{(m) h}$, leading to the conclusion that the generalized first law is also satisfied (as it should by duality) in the electric sector. Another noteworthy consequence of (7.37) is that the electric and magnetic contributions to the generalized dyonic Smarr formula (6.39) are equal, i.e. for dyons of either sector

$$
Q V_{h}=P W_{h}=\frac{\delta^{2} \bar{\beta}}{4 r_{0}(\bar{\beta}+\bar{\delta})} .
$$

\section{Conclusion}

We have shown that the non-asymptotically flat static black hole solutions of EMD theory may be obtained as near-horizon limits of asymptotically flat black holes, and recalled how their mass and angular momentum may be computed unambiguously in the quasilocal energy framework.

We have then concentrated on the special case with dilaton coupling constant $\alpha^{2}=3$, which is a dimensional reduction of five-dimensional KaluzaKlein theory. We have shown that in this case the action of the group $S L(3, R)$ of invariance transformations may be used to generate from the NAF magnetostatic or electrostatic black hole solutions two classes of NAF 
rotating dyonic black hole solutions. The NAF black holes of the magnetic class turn out to be simply dimensional reductions of the AF five-dimensional Myers-Perry black holes relative to one of the azimuthal angles, and their four-dimensional quasi-local mass, quasi-local angular momentum, and "dyonic momentum" (the product of their electric and magnetic charges) may be obtained from the mass and the two angular momenta of the parent fivedimensional black hole by dimensional reduction. We view this as a quite non-trivial success of the quasi-local energy approach, insofar as the computation of the quasilocal mass and angular momentum is rather involved.

On the other hand, the black holes of the electric class are unusual, twisted dimensional reductions of the AF five-dimensional Rasheed black holes with a NUT charge balancing the magnetic charge. It is important to note that the extension of the local "twist" coordinate transformation to a global coordinate transformation introduces a periodicity in time if the fifth dimension is compactified on the Klein circle, so that the NAF black holes of the electric sector are only locally, not globally, equivalent to AF Rasheed black holes. A by-product of this analysis is that, by electro-magnetic duality, there is a oneto-one local correspondence between the Myers-Perry and Rasheed classes of AF five-dimensional black holes.

We have also shown that the first law of black hole thermodynamics, as generalized to dyonic black holes by Rasheed, is satisfied by both classes of NAF rotating dyonic black holes, provided the overall scale parameter $r_{0}$ is not varied. Again, this validity of the first law under variation of three independent parameters is by no means trivial, and constitutes another test of the validity of our quasilocal energy computations. The NAF dyonic black holes are also found to obey a generalized Smarr-like formula, Eq. (6.39).

Finally, we have discussed the construction of NAF multi-extreme black hole solutions. In the static case with generic $\alpha$, these are actually singular multi-center solutions. In the case $\alpha^{2}=3$, we have shown that, besides these, there are also two classes (magnetic and electric) of regular, intrinsically dyonic multi-extreme black hole solutions given by (6.16) and either (6.17)(6.18) or (7.30)-(7.31).

It may be expected that higher-dimensional generalizations of these NAF black holes to NAF static black brane solutions to the Einstein- $p$-form-dilaton theory also exist, as well as NAF rotating and dyonic black branes for special values of the dilaton coupling constant. We intend to address this question in a future publication [45]. 


\section{Appendix A}

Here we derive from the trivially embedded Taub-NUT solution a NUTted NAF black hole of the electric sector, and show that its magnetic dual is a twisted dimensional reduction of the Myers-Perry black hole with two equal angular momenta. The trivial five-dimensional embedding of the Taub-NUT metric is

$$
d s_{5}^{2}=-\frac{\Delta}{\Sigma}\left(d t^{\prime}+2 l \cos \theta d \varphi\right)^{2}+\frac{\Sigma}{\Delta}\left(d r^{2}+\Delta d \Omega_{2}^{2}\right)+\left(d x^{\prime 5}\right)^{2},
$$

with

$$
\Delta=r^{2}-2 m r-l^{2}, \quad \Sigma=r^{2}+l^{2}
$$

The twist

$$
t^{\prime}=\gamma^{-1}\left(t-\gamma^{2} x^{5}\right), \quad x^{\prime 5}=\gamma x^{5},
$$

leads to the five-dimensional metric

$$
\begin{aligned}
d s_{5}^{2}= & -\frac{\Delta}{\Pi}(d t+2 \gamma l \cos \theta d \varphi)^{2}+\frac{\Sigma}{\Delta}\left(d r^{2}+\Delta d \Omega_{2}^{2}\right) \\
& +\frac{\Pi}{\Sigma}\left(d x^{5}+\frac{\Delta}{\Pi}[d t+2 \gamma l \cos \theta d \varphi]\right)^{2},
\end{aligned}
$$

with

$$
\Pi=2 \gamma^{2}\left(m r+l^{2}\right) .
$$

After dimensional reduction, this leads to the four-dimensional NAF "electric" solution

$$
\begin{aligned}
d s_{4}^{2} & =-\frac{\Delta}{\sqrt{\Pi \Sigma}}(d t+2 \gamma l \cos \theta d \varphi)^{2}+\sqrt{\Pi \Sigma}\left(\frac{d r^{2}}{\Delta}+d \Omega_{2}^{2}\right), \\
\mathcal{A} & =\frac{\Delta}{2 \Pi}(d t+2 \gamma l \cos \theta d \varphi), \\
\mathrm{e}^{2 \phi / \sqrt{3}} & =\sqrt{\frac{\Sigma}{\Pi}}
\end{aligned}
$$

The four-dimensional "magnetic" dual solution has the same four-dimensional metric with electromagnetic and dilaton fields

$$
\begin{aligned}
\mathcal{A} & =-\frac{\gamma}{\Sigma}\left(l(r-m) d t+\frac{\gamma m \Lambda}{\Gamma} \cos \theta d \varphi\right) \\
\mathrm{e}^{2 \phi / \sqrt{3}} & =\sqrt{\frac{\Pi}{\Sigma}} \quad\left(\Lambda=r^{2}+2 l^{2} r / m-l^{2}\right) .
\end{aligned}
$$


The corresponding five-dimensional lift

$$
\begin{aligned}
d s_{5}^{2}= & -\frac{\Delta}{\Gamma}(d t+2 \gamma l \cos \theta d \varphi)^{2}+\frac{\Pi}{\Delta}\left(d r^{2}+\Delta d \Omega_{2}^{2}\right) \\
& +\frac{\Sigma}{\Pi}\left(d x^{5}-2 \frac{\gamma}{\Sigma}\left[l(r-m) d t+\frac{\gamma m \Lambda}{\Gamma} \cos \theta d \varphi\right]\right)^{2},
\end{aligned}
$$

may be rearranged as

$$
\begin{aligned}
& d s_{5}^{2}=-(d t+\bar{a} d \eta)^{2}+\frac{\mu}{\rho^{2}}\left(d t+\bar{a} d \eta-\frac{\bar{a}}{2}(d \eta-\cos \theta d \varphi)\right)^{2} \\
& +\frac{\rho^{4}}{\rho^{4}-\mu \rho^{2}+\mu \bar{a}^{2}} d \rho^{2}+\frac{\rho^{2}}{4}\left(d \theta^{2}+d \varphi^{2}+d \eta^{2}-2 \cos \theta d \varphi d \eta\right)
\end{aligned}
$$

with

$$
\rho^{2}=8 \gamma^{2}\left(m r+l^{2}\right), \eta=\frac{1}{2 m \gamma^{2}} x^{5}, \mu=16 \gamma^{2}\left(l^{2}+m^{2}\right), \bar{a}=2 \gamma l .
$$

After carrying out the inverse twist

$$
t=t^{\prime}-\bar{a} \eta
$$

we recognize in (A.12) another form of the Myers-Perry metric with two equal angular momenta, Eq. (5.27) with $a_{+}=a_{-}=\bar{a}$. 


\section{References}

[1] K.C.K. Chan, J.H. Horne and R.B.Mann, Nucl. Phys. B 447 (1995) 441.

[2] G. Clément, D. Gal'tsov and C. Leygnac, Phys. Rev. D 67 (2003) 024012.

[3] R.G. Cai, J.Y. Ji and K.S. Soh, Phys. Rev. D 57 (1998) 6547.

[4] R.G. Cai and Y.Z. Zhang, Phys. Rev. D 64 (2001) 104015.

[5] O. Aharony, M. Berkooz, D. Kutasov, and N. Seiberg, JHEP 10 (1998) 004.

[6] J.D. Brown and J.W. York, Phys. Rev. D 47 (1993) 1407.

[7] S.W. Hawking and G.T. Horowitz, Class. Quant. Grav. 13 (1996) 1487.

[8] C.M. Chen and J. Nester, Grav. \& Cosm. 6 (2000) 257.

[9] I.S.N. Booth, A quasilocal hamiltonian for gravity with classical and quantum applications, gr-qc/0008030.

[10] J.M. Bardeen, B. Carter and S.W. Hawking, Commun. Math. Phys. 31 (1973) 161.

[11] M.I. Cai and G.J. Galloway, Class. Quantum Grav. 18 (2001) 2707.

[12] G. Gibbons and D. Wiltshire, Ann. Phys. (N. Y.) 167 (1986) 201.

[13] D. Rasheed, Nucl. Phys. B 454 (1995) 379.

[14] F.R. Tangherlini, Nuovo Cimento 77 (1963) 636.

[15] R. Myers and M. Perry, Ann. Phys. (N.Y.) 172 (1986) 304.

[16] R. Emparan and H.S. Reall, Phys. Rev. Lett. 88 (2002) 101101.

[17] G.W. Gibbons and K. Maeda, Nucl. Phys. B 298 (1988) 741.

[18] D. Garfinkle, G.T. Horowitz and A. Strominger, Phys. Rev. D 43 (1991) 3140.

[19] A.K.M. Masood-ul-Alam, Class. Quantum Grav. 10 (1993) 2649. 
[20] M. Mars and W. Simon, On uniqueness of static Einstein-Maxwelldilaton black holes, gr-qc/0105023.

[21] S.B. Giddings and A. Strominger, Phys. Rev. D 46 (1992) 627.

[22] M. Gürses and E. Sermutlu, Class. Quantum Grav. 12 (1995) 2799.

[23] Y. Kiem and D. Park, Nucl. Phys. B 486 (1997) 114.

[24] M. Bañados, C. Teitelboim and J. Zanelli, Phys. Rev. Lett. 69 (1992) 1849; M. Bañados, M. Henneaux, C. Teitelboim and J. Zanelli, Phys. Rev. D 48 (1993) 1506.

[25] D.V. Galtsov, A.A. Garcia and O.V. Kechkin, Class. Quantum Grav. 12 (1995) 2887.

[26] G. Neugebauer and D. Kramer, Ann. Phys. (Leipzig) 24 (1969) 62.

[27] H. Stephani, D. Kramer, M. MacCallum, C. Hoenselaers and E. Herlt, "Exact Solutions to Einstein's Field Equations", 2nd edition (Cambridge University Press, Cambridge, 2003).

[28] G. Clément, Gen. Rel. Grav. 18 (1986) 861

[29] G. Clément, Phys. Lett. A 118 (1986) 11.

[30] G. Clément and D. Gal'tsov, Phys. Rev. D 54 (1996) 6136.

[31] G.W. Gibbons, D. Kastor, L.A.J. London, P.K. Townsend and J. Traschen, Nucl. Phys. B 416 (1994) 850.

[32] R. Kallosh and A. Peet, Phys. Rev. D 46 (1992) 5223.

[33] R. Arnowitt, S. Deser and C.W. Misner, The Dynamics of General Relativity, in "Gravitation: An Introduction to Current Research", ed. L. Witten (Wiley, New York, 1962).

[34] G. Clément, Phys. Rev. D 68 (2003) 024032.

[35] D.V. Gal'tsov, Phys. Rev. Lett. 74 (1995) 2863.

[36] B. Jensen and U. Lindström, Phys. Rev. D 52 (1995) 3543. 
[37] G. Clément, Phys. Rev. D 57 (1998) 4885.

[38] G. Clément, Grav. \& Cosm. 5 (1999) 281.

[39] D. Maison, Gen. Rel. Grav. 10 (1979) 717.

[40] D.J. Gross and M.J. Perry, Nucl. Phys. B 226 (1983) 29.

[41] W.A. Israel and G.A. Wilson, J. Math. Phys. 13 (1972) 865; Z. Perjès, Phys. Rev. Lett. 27 (1971) 1668.

[42] S. Deser, R. Jackiw and Gerard 't Hooft, Ann. Phys. (NY) 152 (1984) 220.

[43] S.W. Hawking and S.F. Ross, Phys. Rev. D 52 (1995) 5865.

[44] G.W. Gibbons, Nucl. Phys. B 207 (1982) 337.

[45] G. Clément, D. Gal'tsov and C. Leygnac, in preparation. 


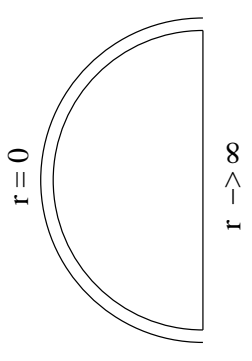

$(\mathrm{b}<0)$

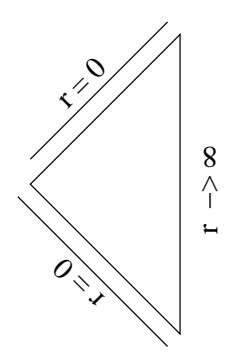

$(b=0)$

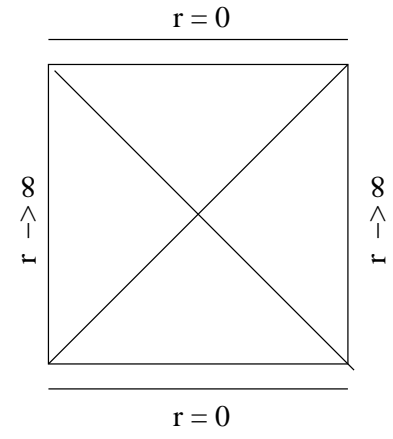

$(\mathrm{b}>0)$

Figure 1: Penrose diagrams of (2.8) for $0<\alpha<1$ with $b<0, b=0$ and $b>0$.

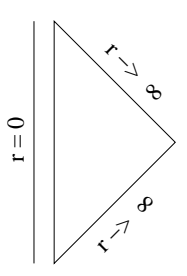

$(\mathrm{b}<0)$

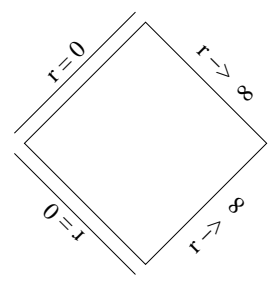

$(\mathrm{b}=0)$

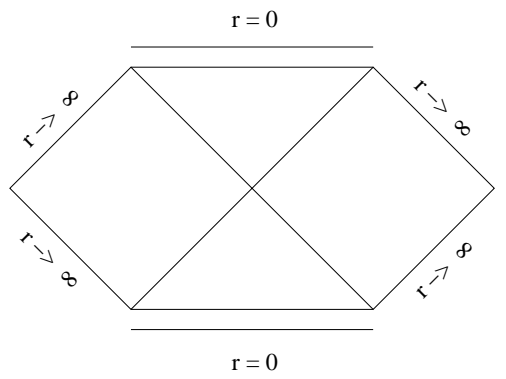

$(\mathrm{b}>0)$

Figure 2: Penrose diagrams of (2.8) for $\alpha=1$ with $b<0, b=0$ and $b>0$. 


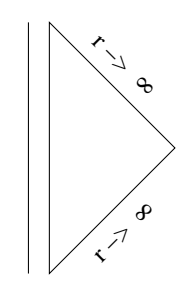

$(\mathrm{b}<0)$

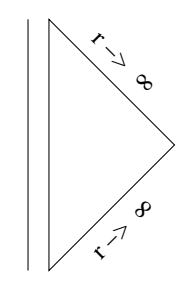

$(b=0)$

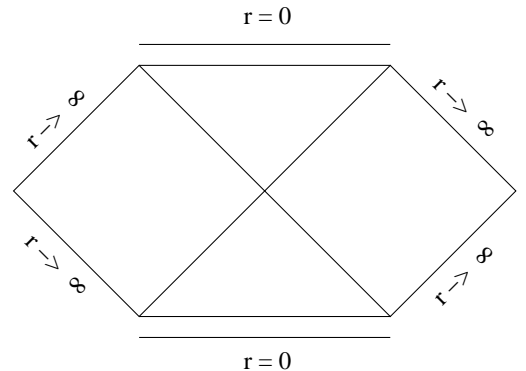

$(\mathrm{b}>0)$

Figure 3: Penrose diagrams of (2.8) for $\alpha>1$ with $b<0, b=0$ and $b>0$. 